2. González Álvaro I, Estévez del Toro M, Nieto S, García de Vicuña R, Castañeda S, García-Vadillo A. Amiloidosis secundarias en espondiloartropatías seronegativas. Rev Esp Reumatol 1994; 21: 241-4.

3. Rodney H. Falk M.D, Raymond L, Comenzo M.D, Martha Skinner, M.D. The systemic Amyloidises. N Engl J Med 1997; 337: 898-909.

4. Sanmartí R, Muñoz J. Amiloidosis. Medicine 2001; 8 (35): 1709-14.

5. Muñoz Gómez, J.Amiloidosis secundaria en las enfermedades reumáticas. Sem Reumatol 2000; 1: 225-31.

6. Ahmed Q, Chung-Park M, Mustafa K, Khan MA. Psoriatic spondyloarthropathy with secondary amyloidosis. J Rheumatol 1996;23 (6): 1107-10.

7. Romero Yuste S. Artritis psoriásica. Medicine 2001; 8 (31): 1612-5.

8. Sanmartí Sala R, Muñoz Gómez J. Amiloidosis. Medicine 2001; 8 (33): 1709-14

9. Muñoz Gómez J. Diagnóstico y seguimiento de la amiloidosis. Rev Esp Reumatol 1996; 23: 21-5.

10. Garrido Serrano A, Guerrero Igea FJ, Hierro Guilmain C, Ruiz Lupianez E, Palomo Gil S. Respuesta favorable a la colchicina en la amiloidosis secundaria a la enfermedad inflamatoria intestinal. Gastroenterol Hepatol 2001; 24 (4): 196-8.

11. Kagan A, Husza'r M, Frumkin A, Rapoport J. Reversal of nephrotic syndrome due to AA amyloidosis in psoriatic patients on long-term colchicine treatment. Case report and review of the literature. Nephron 1999; 82 (4): 348-53.

\title{
Efectos adversos originados por la inserción de un pleurecath
}

\section{Señor Director:}

La inserción de un drenaje torácico mediante el empleo de un pleurecath es un procedimiento invasivo escasamente asociado a complicaciones, inferior al 1\% (1-3). Describimos un caso de neumomediastino asociado a la inserción de un catéter pleural de fino calibre.

Paciente de 55 años que sufre traumatismo torácico y pélvico tras una caída desde 5 metros. En el lugar del accidente presenta GCS 15 e insuficiencia respiratoria con hipoventilación del pulmón izquierdo por lo que se implanta un pleurecath. Es ingresado en UCI donde se practica Rx de tórax en la que se aprecian fracturas costales izquierdas múltiples, presencia de neumomediastino, contusión pulmonar bilateral y existencia de un catéter que parece llegar hasta pericardio. En la TAC pulmonar con contraste practicada de modo urgente, además de lo mencionado se objetiva que dicho tubo se encuentra adyacente al margen del cayado aórtico (Fig. 1). Tras retirada de este drenaje el paciente es dado de alta tras 72 horas de permanencia en UCI.

El empleo de técnicas aún aparentemente sencillas como la implantación de un drenaje torácico mediante dispositivos como el pleurecath, debe hacernos valorar además de la indicación de su inserción, la práctica de los mismos por personal especializado y entrenado en aras de evitar la presentación de efectos adversos, que en el caso expuesto podría haber precisado una intervención quirúrgica urgente de la aorta, o bien el fallecimiento del paciente en el lugar de los hechos $(4,5)$.

\section{B. Obón-Azuara, I. Gutiérrez-Cía, J. L. Ruiz-Gurbindo}

Servicio de Medicina Intensiva. Hospital Clínico Universitario. Zaragoza

1. Valette H, Giustozzi A. Another complication of thoracic drainage: Perforation-intubation of the subclavian vein. J Chir 1996;133: 91-2.

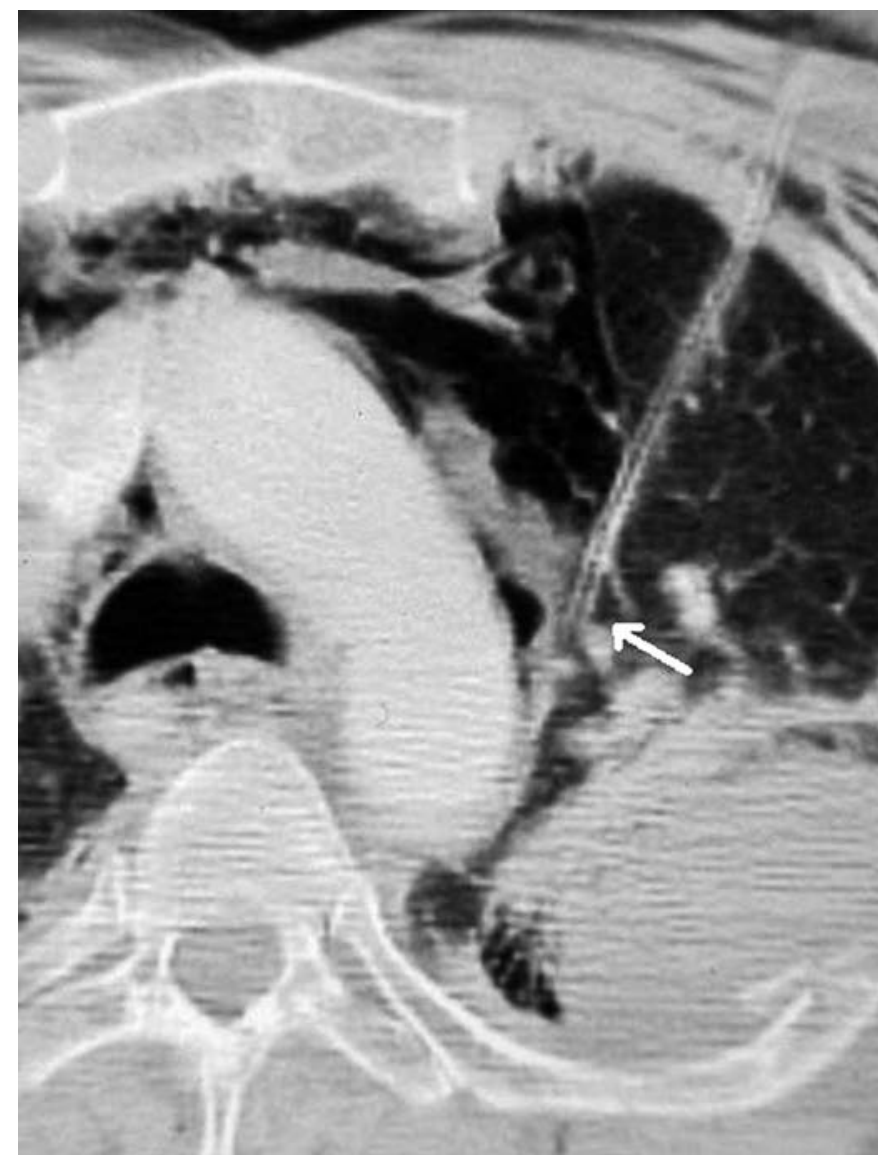

Fig. 1. TAC torácica: Catéter situado adyacente al cayado aórtico.

2. Kerger H, Blaettner T, Froehlich C, Erns J, Friestch T, Isselhorst C, Nguyen AK, Volz A, Fiedler F, Genzwuerker HV. Perforation of the left atrium by a chest tube in a patient with cardiomegaly: Management of a rare, but life-threatening complication. Resuscitation. 2007. Epub ahead of print.

3. Shih CT, Chang Y, Lai ST. Successful management of perforating injury of right atrium by chest tube. Zhonghua Yi Xue Za Zhi (Taipei) 1992; 50: 338-40.

4. Colt HG, Brewer N, Barbur E. Evaluation of patient-related and procedure-related factors contributing to pneumothorax following thoracentesis. Chest 1999; 116: 134-8

5. Needham DM, Sinopoli DJ, Thompson DA, Holzmueller CG, Dorman T, Lubomski LH, Wu AW, Morlock LL, Makary MA, Pronovost PJ. A system factors analysis of "line, tube, and drain" incidents in the intensive care unit. Crit Care Med 2005; 33: 1701-7.

\section{Cadenas ligeras libres en suero. Utilidad clínica}

\section{Sr. Director:}

Se publicó un trabajo en su revista de Molina et al. (1) y nos gustaría comentar que un nuevo parámetro, la determinación de la concentración de cadenas ligeras libres en suero (CLLs), ya es una realidad en la práctica clínica, lo que supone un avance en el diagnóstico y seguimiento de las gammapatías monoclonales.

La mayor parte de las cadenas ligeras del organismo se encuentran asociadas con cadenas pesadas para dar lugar a los 
anticuerpos. No obstante, existe una pequeña fracción, formada por cadenas de tipo $\kappa$ y $\lambda$ en proporción constante, que circula libre en condiciones fisiológicas. Las alteraciones de su concentración $y$, sobre todo, del cociente $\kappa / \lambda$, son características de las enfermedades causadas por la proliferación incontrolada de un clon de célula plasmática, en las que hay una gran producción de cadenas ligeras libres, pero sólo del tipo secretado por el clon proliferante, ya sea $\kappa$ o $\lambda$.

La electroforesis de proteínas en suero (EPS) y la inmunofijación (IFE) son los métodos analíticos habitualmente empleados para detectar la presencia de CLLs. Sin embargo son poco sensibles y no siempre proporcionan resultados cuantitativos. Estos inconvenientes han quedado resueltos con el desarrollo de nuevos inmunoensayos, cuya sensibilidad es mayor y además permiten cuantificar con precisión las CLLs. Gracias a esto ahora es posible hacer un diagnóstico precoz en aquellos casos que cursan con una producción muy baja de inmunoglobulinas.

Las CLLs no están aumentadas en todas la gammapatías monoclonales, por lo que su determinación debe enfocarse como un test complementario, sin excluir la realización de otras pruebas convencionales. Son particularmente interesantes en dos situaciones: por una parte, cuando un paciente presenta indicios de estar sufriendo un proceso neoplásico de células plasmáticas sin confirmación por EPS o IFE, lo que ocurre con frecuencia en casos de mieloma múltiple no secretor, mieloma múltiple de cadenas ligeras (mieloma de Bence Jones) y amiloidosis sistémica. De otro lado, en las gammapatías monoclonales de significado incierto (GMSI) el cociente $\kappa / \lambda$ ha demostrado ser un factor de riesgo independiente de la progresión a gammapatía maligna (3).

Los valores de referencia en personas sanas definidos por Katzmann et al (4) son: CLLs $\kappa=3,3-19,4 \mathrm{mg} / \mathrm{l}$; CLLs $\lambda=5,7-$ $26,3 \mathrm{mg} / \mathrm{l}$; ratio $\kappa / \lambda(95 \%$ del intervalo de referencia $)=0,3-1,2, \mathrm{si}$ bien los autores recomiendan establecer unos valores propios en cada laboratorio.

Presentamos un estudio retrospectivo donde analizamos los factores analíticos que demostraron ser predictivos de evolución de las GMSI a gammapatías monoclonales malignas (GMM) en una serie de 618 casos (5) seguidos durante un periodo de 134 meses. Se clasificaron por subtipos: $\operatorname{Ig} A G, \operatorname{Ig} A, \operatorname{IgM}, \operatorname{IgD}$, y por sus cadenas ligeras: $\kappa$ o $\lambda$. Se analizó cuántas evolucionaron a proceso maligno y se halló que hubo veintiocho casos de transformación a GMM y cuatro síndromes linfoproliferativos crónicos (tres Linfomas No Hodkin-B del manto y una Leucemia linfoide crónica). Los factores analíticos asociados a GMM y procesos linfoproliferativos fueron:

- Cadena pesada IgA $(\mathrm{p}<0,002)$

- VSG $(p<0,0001)$.

- Porcentaje de células plasmáticas en médula ósea $(\mathrm{p}<0,002)$.

-Componente monoclonal.

Aunque en la serie no se calculó el cociente de cadenas libres en suero basándonos en diversos trabajos $(3,6)$ proponemos su inclusión en la práctica diaria para el seguimiento de un pico monoclonal, con el objetivo de identificar pacientes de alto y bajo riesgo, de tal modo que se pueda intensificar el seguimiento de los primeros y evitar pruebas innecesarias a los segundos.

\section{A. Encinas Madrazo, M. E. González García ${ }^{1}$, J. Cepeda Piorno, A. J. González Huerta, E. Fernández Rodríguez}

\section{Servicios de Análisis Clínicos y 'Hematología. Hospital de} Cabueñes. Gijón, Principado de Asturias

1. Molina Garrido MJ, Guillén Ponce C, Guirado-Risueño M, Martínez y Sevila C, Carrato Mena A. Diagnóstico diferencial de las gammapatías monoclonales. An Med Interna (Madrid) 2006; 23: 546-51.

2. Lachmann HJ, Gallimore R, Gallimore JD, Carr-Smith HD, Bradwell
AR, Pepys MB, Hawkins PN. British Journal of Haematology: Outcome in systemic AL amyloidosis in relation to changes in concentration of circulating free immunoglobulin light chains following chemotherapy. Brit J Haematol 2003; 122: 78-84.

3. Rajkumar SV, Kyle RA, Therneau TM, Melton LJ III, Bradwell AR, Clark RJ, Larson DR, Plevak Mf, Dispenzieri A, Katzmann JA. Serum free light chain ratio is an independent risk factor for progression in monoclonal gammopathy of undetermined significance. Blood 2005 : 106: $812-817$

4. Katzmann JA, Clark RJ, Abraham RS, Bryant S, Lymp JF, Bradwell $\mathrm{AR}$, Kyle RA. Serum reference intervals and diagnostic ranges for free $\kappa$ and free $\lambda$ inmunoglobuline light chains: relative sensitivity for detection of monoclonal light chains. Clin Chem 2002; 48: 1437-44.

5. González García ME, Fernández Álvarez C, Robles Marinas V, González Huerta AJ, González Rodríguez AP, García Fernández J. Incidente of monoclonal gammopathy of undetermined significance in sanitary area. Haematologica 2007; 1 (Supl. 2): 78

6. Katzmann JA, Abraham RS, Dispenzieri A, Lust JA, Kyle RA. Diagnostic performance of quantitative $\kappa$ and $\lambda$ free light chain assays in clinical practice. Clin Chem 2005; 51: 878-81.

\section{Velocidad de sedimentación "extrema" vs. proteína $\mathrm{C}$ reactiva}

\section{Sr. Director:}

La velocidad de sedimentación globular es una determinación analítica de amplio uso en la práctica clínica aunque muy poco específica. Su utilidad clínica como screening de enfermedad es cuestionable, sin embargo valores superiores a 100 son significativos y están asociados a patología. Rara vez se objetivan valores mayores a 150. A continuación describimos un caso con cifras de VSG de $202 \mathrm{~mm} / \mathrm{h}$.

Una mujer de 80 años, independiente en autocuidado (Índice de Barthel 100) y con antecedentes personales: hipoacusia bilateral, fractura de cadera derecha intervenida mediante enclavado intramedular, prótesis total de cadera izquierda hace 5 años por coxartrosis y diagnosticada de mieloma múltiple III A Ig M cadenas Kappa habiendo recibido previamente distintos ciclos de melfalán y prednisona y con cifras de VSG cuatro meses antes del ingreso de $111 \mathrm{~mm} / \mathrm{h}$ e Ig M de $2.784 \mathrm{mg} / \mathrm{dl}$. Acude a Urgencias por presentar exudado por cadera izquierda de 1 mes de evolución. Al ingreso se determinan entre otras pruebas VSG obteniendo valores de $202 \mathrm{~mm} / \mathrm{h}$, beta 2 microglobulina 2291 (600-2.700) y PCR de 5,2 mg/dl (0-0,5). Se utilizó el analizador Sedimatic 100. Se obtuvieron cultivos del exudado que fueron negativos, se realizó un TAC de cadera izquierda objetivando colección 4 x $2 \mathrm{~cm}$ y ecografía compatible con absceso yuxtaoseo fistulizado a piel. Se instauró tratamiento empírico con Teicoplanina, con buena evolución clínica y analítica (no toleró Rifampicina por vómitos), presentando al alta una VSG de $165 \mathrm{~mm} / \mathrm{h}$ y PCR de $1,2 \mathrm{mg} / \mathrm{dl}$.

La velocidad de sedimentación globular es un test habitual de laboratorio descubierto a finales de 1700 (1) y basado en la mayor sedimentación de los glóbulos rojos objetivada en el laboratorio en muchas enfermedades. Se ha utilizado para cuantificar el proceso inflamatorio que acompaña a enfermedades inflamatorias, infecciosas y neoplásicas.

Hay estudios en enfermos comunitarios donde se objetivó una prevalencia de VSG mayor a 100 del 4,2\%, siendo la causa infecciosa la más frecuente (33\%), neoplasia maligna (17\%), enferme- 